\title{
Effects induced by the passage of the Sun through dense molecular clouds
}

\section{Flow outside of the compressed heliosphere}

\author{
A. Yeghikyan ${ }^{1,2}$ and H. Fahr ${ }^{1}$ \\ ${ }^{1}$ Institute of Astrophysics and Extraterrestrial Research (IAER), University of Bonn, Auf dem Huegel 71, 53121 Bonn, \\ Germany \\ 2 Byurakan Astrophysical Observatory, Armenia
}

Received 18 August 2003 / Accepted 7 November 2003

\begin{abstract}
The problem of passages of the Solar System through dense, dusty and cold interstellar gas clouds is discussed. A considerable number of such encounters must have already occurred in the past and these events must have pushed the heliosphere inward to the region of the terrestrial planets, provided a number density of the cloud's material of the order of, or greater than, $1000 \mathrm{~cm}^{-3}$, and relative velocities of about $20 \mathrm{~km} \mathrm{~s}^{-1}$ prevail. Under these conditions it will happen that the Earth is directly exposed to the interstellar flow. Neutral interstellar hydrogen and proton densities at the Earth's orbit are calculated under conditions of the heliosphere with the heliopause reduced to dimensions smaller than 1 AU. A simple twofluids treatment of the incoming flow, ionized by the solar UV only, is proposed here and the amount of neutral interstellar hydrogen is assessed which is accreted by the Earth's atmosphere during one passage. The behavior of the flow variables is investigated by a 2D-hydrodynamic approach to model the interaction processes. Because of a reduction of the heliospheric dimension down to $1 \mathrm{AU}$, direct influence of the cloud's matter to the terrestrial environment and atmospheric chemistry must be envisaged. This will be briefly discussed in the paper.
\end{abstract}

Key words. ISM: clouds, solar wind - hydrodynamics, earth

\section{Introduction}

The interstellar medium (ISM) in our Galaxy, a mixture of plasma, gas and dust, is known to be highly inhomogeneous with respect to density and temperature. It tends to concentrate near the Galactic plane and along the spiral arms and appears as a dense, neutral and molecular material, embedded in an ambient warm and tenuous medium (for an overview of the composition of the ISM, see McKee 1995). Roughly half the ISM mass is confined to discrete clouds occupying only $\sim 1 \div 2 \%$ of the Galactic disk volume. The dense component consists of cold atomic $\mathrm{H}$ I clouds $(T \sim 100 \mathrm{~K})$ and giant molecular clouds (GMC) $(T \sim 10 \div 20 \mathrm{~K})$ with a clumpy substructure. These clouds have irregular shapes and overall dimensions ranging from $0.01-50 \mathrm{pc}$ and densities from $10-100 \mathrm{~cm}^{-3}(\mathrm{HI})$ and of about $1000 \mathrm{~cm}^{-3}$ (GMC clouds), respectively. Cores of GMC and clumps within GMC are even denser (more than 1000 up to $10^{5} \mathrm{~cm}^{-3}$ ), but they occupy only $\sim 0.1$ and $\sim 1 \%$ of the GMC volume, respectively (Allen 2000).

The possibility of solar encounters with dense interstellar clouds (IC) with particle concentrations about $10 \div 1000 \mathrm{~cm}^{-3}$

Send offprint requests to: A. Yeghikyan,

e-mail: aray@astro.uni-bonn.de and more, is of great interest in view of its possible effects upon the Earth. The question of whether dense IC would prevent the solar wind (SW) from reaching the Earth with the result of cloud material directly impacting onto the terrestrial atmosphere, as well as many other aspects of this complex problem, were already discussed in the literature of the past (Fahr 1968a,b; Talbot \& Newman 1977; Holzer 1977; Ripken \& Fahr 1981; Zank \& Frisch 1999; Scherer 2000; Scherer et al. 2002; Frisch et al. 2002 and references therein). Such a scenario is considered as possibly triggering global glaciations, depositions of a prebiotic material on the primordial Earth, possible ecological repercussions for the Earth due to an accretion of the cloud's matter, and of course, bio-mass extinction (Yabushita \& Allen 1997).

It is straightforward to estimate that neutral $\mathrm{H}$ I clouds, having a mean density of 10 to a few $100 \mathrm{~cm}^{-3}$, and a radius of about a few pc, are objects frequently encountered by the Sun during its galactic journey (more than 100 times since its birth $\sim 4.6$ Gyr ago), while even the more dense GMC's must have been encountered at least a few times (Yeghikyan \& Fahr 2003 and references therein).

Nevertheless Yabushita \& Allen $(1983,1989,1997)$ have pointed out that just such rare passages through the cores of 
the GMC's may have happened and have the effects of substantially depleting the oxygen of the Earth's atmosphere and e.g. causing the bio-mass extinction at the Cretaceous/Tertiary boundary. Furthermore only such a cataclysmic (not catastrophic) event is compatible with the well known high concentration of iridium in a clay layer at this boundary, $65 \mathrm{Myr}$ ago, whereas cometary impacts could not have deposited enough iridium-containing material. Finally, Wimmer-Schweingruber \& Bochsler (2000) have recently interpreted an implanted gas in surface layers of lunar soil grains as a record due to the encounter with a dense interstellar cloud.

To investigate the behavior of neutral matter inside the heliopause, which is a separatrix between the solar wind and the ambient interstellar medium, the interaction of the solar wind with the countermoving flow usually is treated as the problem of the penetration of neutral atoms from the local ISM (LISM) into the heliosphere (see Fahr 2003 for a recent review).

It is clear that an influence of both gravity and photoionization on the neutral hydrogen flow into the heliosphere should be important for quantitative determinations of the flow parameters at regions near the Earth's orbit (Yeghikyan \& Fahr 2003). The prevailing physical conditions in the reduced heliosphere suggest that the idealized hydrodynamic equations with the inclusion of effects of gravity and ionization can be used here (Yeghikyan \& Fahr 2003). To the contrast, the kinetic treatment requires very time-consuming numerical codes and we therefore prefer a hydrodynamic modelling here, which is justified for IC densities of, and greater than, a few $100 \mathrm{~cm}^{-3}$ (see e.g. Yeghikyan \& Fahr 2003). Thus, to describe the behavior of the flow outside of the heliosphere, here reduced to regions inside of $1 \mathrm{AU}$, one may use a simple gasdynamic interaction model, where the system of fluid equations for neutral material and solar wind plasma are dynamically decoupled (e.g. see Whang 1996), but for the outer flow gravity and, instead of charge exchange between $\mathrm{H}$-ions and $\mathrm{H}$-atoms, photoionization is taken into account here.

In this paper we use the closed system of fluid Euler equations to calculate a $2 \mathrm{D}$ gasdynamic time-dependent simulation of neutral and ionized flow parameters when the heliosphere is drastically reduced down to a dimension of about $1 \mathrm{AU}$ or less (Yeghikyan \& Fahr 2003), as consequence of the increased LISM-ram pressure connected with a density of the countermoving IC material, i.e. 100-1000 times greater than at present. According to the above mentioned estimates the Sun during its life has encountered such HI clouds more than one hundred times, while only a few denser GMC's (i.e. $n_{\mathrm{i}}>10^{3} \mathrm{~cm}^{-3}$ ) may have been met.

Not included in the present model are external magnetic field influences on the heliospheric structure; but it turns out that even at a 10-fold increased field strength (Heiles et al. 1991) its magnetic pressure cannot be comparable to the dynamic pressure of the IC resulting at a moderate density $n=$ $100 \mathrm{~cm}^{-3}$ (Parker 1963).

It should be noted here, that in its early history, the Sun was probably more active than it is now (Guinan \& Ribas 2002 and references therein). To be more correct, the Sun, some $4.6 \mathrm{Gyr}$ ago, had an initial luminosity of $\sim 70 \%$ of the present Sun and was about $200 \mathrm{~K}$ cooler, but perhaps had a UV enhancement with respect to the present day UV irradiation in the range $\approx 150-250 \mathrm{~nm}$ by a factor 2 during the first few $10^{8} \mathrm{yr}$ (Zahnle $\&$ Walker 1982). The values for the earlier solar wind intensity are more uncertain. They are based on observational studies of stellar winds from young solar type T-Tauri stars with different ages and indicate that the winds of the young Sun could possibly have been 100-1000 times more intense than at the present epoch (Lammer et al. 2002). Finally, Sackmann \& Boothroyd (2003) quite recently have shown that the current accurate helioseismic data are consistent with high-precision solar evolutionary models, which predict a more luminous Sun with a stronger wind than envisaged by the standard model at the first 1-2 Gyr after the origin of the Sun. Such powers (23 orders of magnitude) of the earlier solar wind encountering with HI cloud would have enlarged the heliosphere to beyond 10 AU and greatly caused an increase of the ionization degree of neutral matter at $1 \mathrm{AU}$. Dense GMC probably would be able to suppress the powerful solar wind inside $1 \mathrm{AU}$ due to stronger dynamic counterpressures.

In the following calculations we will adopt the present parameters of the solar output, which are valid for the most recent events under consideration (encounters with IC during last 23 Gyr), while more specific models should be developed for the more earlier ones.

\section{The adequate view of the interaction with an interstellar cloud}

The encounter of the solar system with a dense interstellar cloud would of course lead to a strongly time-dependent reaction of its extended heliospheric plasma structures as they exist for instance at present. When the extended heliosphere with an upwind extension of about $150 \mathrm{AU}$ will first get into contact with the predominantly neutral gas material of an approaching cloud, then at the first period of the encounter the extended supersonic and subsonic solar wind will be neutralized by heavy charge exchange with incoming neutral $\mathrm{H}$-atoms. As a consequence of this event energetic neutral atoms with energies of the order of $1 \mathrm{keV}$ will be produced which radially run outwards from the heliosphere into the arriving cloud material. By elastic $\mathrm{H}-\mathrm{H}$ collisions these energetic atoms will have strongly heated up the cloud material upon arrival at the heliopause. A strong pressure gradient will thus be produced which on the axis reduces the inflow velocity of the cloud material and pushes the cloud gas away from the stagnation point to the flanks of the heliopause which in addition due to the increasing outer interstellar ram pressure shrinks to very small dimensions.

Here we do not intend to consistently describe this strongly time-dependent adaptation history, but we shall presume that eventually a nearly stationary counterflow configuration will have established with the main cloud material becoming deviated around a strongly reduced heliopause (see illustrative representation in Fig. 1). This resulting heliopause configuration we predefine by assuming that it is determined by pressure equilibrium between the strongly coupled inner solar wind plasma flow and the outer interstellar flow constituted by ionized cloud material. 


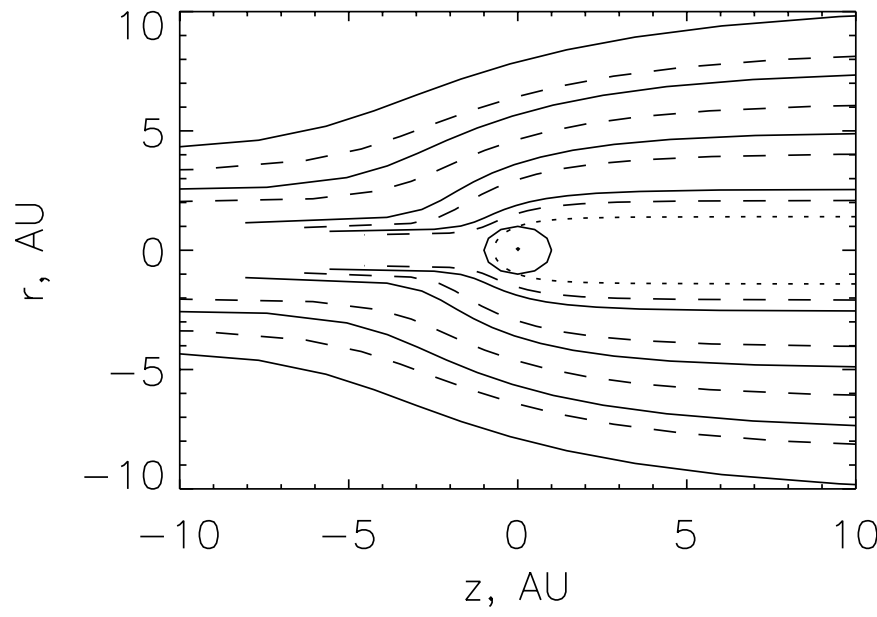

Fig. 1. Qualitative view of the streamlines of the neutral (solid lines) and ionized (dashed lines) flow of the IC wind in the presence of the solar wind, compressed in the upwind direction to inside of $1 \mathrm{AU}$. The Sun is in the origin of the coordinate system. The heliopause is shown by the dotted line and the Earth's orbit (shown by solid circle) is outside of it in the upwind direction.

As is evident from arguments given in the previous section, neutral interstellar cloud matter, when approaching the Solar System, systematically becomes more ionized by photoionization due to the solar EUV radiation. On the other hand, at ISM $\mathrm{H}$-atom densities of the order of $n_{\mathrm{H}}=10^{2} \div 10^{3} \mathrm{~cm}^{-3}$ the free passage of a $1 \mathrm{keV}$ proton (i.e. solar wind proton) is restricted by intensive charge exchange reactions to a mean free path of less than $\lambda_{\mathrm{ex}}=\left(n_{\mathrm{H}} \sigma_{\mathrm{ex}}\right)^{-1}=2 \cdot\left(10^{-1} \div 10^{-2}\right)$ AU. At such solar distances of less than $1 \mathrm{AU}$ the interstellar matter is, however, essentially photoionized. Hence at these small distances, in a first-order view, an interaction of the solar wind protons with the ionized component of the ISM, rather than with the neutral ISM component occurs. Adopting an $\mathrm{H}$-ionization degree $\epsilon$ of the ISM at the border of the plasma-plasma interaction configuration, one can thus denote the density of "hydrodynamically" interacting interstellar protons by $n_{\mathrm{p}}=\epsilon \cdot n_{\mathrm{H}}$ and on the basis of a Parker-type plasma-plasma interaction configuration (see Parker 1963) can estimate a corresponding stand-off distance $L$ of the resulting heliopause by (Fahr 2000):

$L^{2}=r_{0} r_{\mathrm{sh}} \sqrt{\frac{\rho_{\mathrm{w} 0}}{4 \epsilon \rho_{\mathrm{H}}}} \frac{V_{\mathrm{w} 0}}{V_{\mathrm{H}}}$.

Here $r_{0}$ is an inner solar reference distance where the unperturbed solar wind has a density $\rho_{\mathrm{w} 0}$ and a velocity $V_{\mathrm{w} 0}$, respectively. The unperturbed ISM is characterized by a mean density $\rho_{\mathrm{H}}$ and a velocity $V_{\mathrm{H}}$ relative to the solar system. $r_{\mathrm{sh}}$ denotes the termination shock distance from the Sun which by physical reasons in any case should be smaller than $L$. The expected termination shock of the solar wind is located at:

$r_{\mathrm{sh}}=r_{0} \sqrt{\frac{3 K(\gamma) \rho_{\mathrm{w} 0}}{4 C_{\mathrm{ISM}}}} V_{\mathrm{w} 0}$,

where $C_{\mathrm{ISM}}$ is the Bernoulli constant of the ionized ISM given by:

$C_{\mathrm{ISM}}=\epsilon \rho_{\mathrm{H}}\left[\frac{1}{2} V_{\mathrm{H}}^{2}+\frac{\gamma-1}{\gamma} \frac{P_{\mathrm{H}}}{\rho_{\mathrm{H}}}\right]$.
Equations (1)-(3) are valid in the subsonic case (i.e. if the Mach number of the relative IC motion is less than 1). Similar, but more complicated expressions for the supersonic motion of the IC one can find in the cited paper Fahr (2000).

Now on the basis of the above heliospheric dimensions and, with the assumption of a Parker-type plasma-plasma interaction configuration, the heliopause geometry is determined by:

$R(\phi)=R_{0} f(\phi)$,

where $R_{0}=L, \phi=\pi-\theta$, and $\theta$ is the angle between the streamline and the direction of the Sun's motion. The function $f(\phi)$ can be modeled based on existing analytical representations for the above addressed configuration (Fahr et al. 1988; Ratkiewicz 1992; Wilkin 1996). We have used the function given by Wilkin (1996),

$R(\phi)=R_{0} \csc \phi[3(1-\phi \cot \phi)]$,

and can now start our calculations assuming that the IC when approaching the Solar System, but yet outside the heliopause, is only subject to solar photoionization processes. Only after passage over the heliopause the neutral ISM H-atom gas will then become strongly subject to charge exchange processes with the solar wind protons. Here in this paper we do not intend to consistently describe this inner region.

Nevertheless we can argue that the size and shape of the heliopause derived with the equations above, practically are not influenced by the pickup ions created from ionization of neutral atoms occurring between the Sun and the heliopause/termination shock. This is due to the fact that $\mathrm{H}$-atoms undergoing charge exchange in the innerheliospheric region with a supersonic solar wind produce H-pick-up ions which not only act upon the solar wind by momentum loading but also by their pressure gradient. It can then be shown (see Fahr \& Rucinski 2002; Fahr 2002; Fahr \& Chashei 2002) that the effect of H-pick-up ions, since behaving nearly isothermal with the solar wind expansion, is just nothing else as to bring these two oppositely directed forces to cancellation and to guarantee an essentially undecelerated solar wind flow and then to keep the ram pressure at a nearly constant level. In this region downstream of the termination shock the charge-exchange processes of H-atoms with solar wind protons anyway are of nearly no dynamical and thermodynamical effects since in this subsonic plasma region H-pick-up ions hardly can be differentiated from original solar wind protons. Taking these two facts together our conclusion thus is that neutral $\mathrm{H}$-atoms penetrating into the inner and outer heliosphere, even though not treated quantitatively here, can be expected nearly not to change the heliospheric configuration compared to that one taken into account here.

\section{Change of the ionization degree and chemical state in the circumsolar flow}

If the Sun were at rest with respect to the LISM, then a stationary state requires that a local equilibrium prevails up to distances where locally photo-dissociation (photo-ionization) and recombination rates cancel. The degree of ionization of the interstellar gas surrounding the Sun at rest has been determined 
for modest densities in earlier works of Williams (1965) and Krasnobaev (1971) using the assumptions, that there is no hydrodynamic motion of the LISM and effects of the solar wind are negligible. These authors have shown similar results for the density range $1 \mathrm{~cm}^{-3} \leq n \leq 500 \mathrm{~cm}^{-3}$ : gradual decrease of the ionization degree with solar distance up to a few $1000 \mathrm{AU}$, in accordance with a simple estimation of the thickness of the transition region, $d \sim(a \cdot n)^{-1}$, where $a=6 \times 10^{-18} \mathrm{~cm}^{2}$ is the photoionization cross section of HI. In more dense molecular environments, existing in the cores of GMC and their clumpy substructures, with densities of about $10^{5} \mathrm{~cm}^{-3}$, an analogous photodissociation region should be developed due to the fact that photodestruction of the $\mathrm{H}_{2}$ is produced by photon absorption in UV lines by, $2 \div 3$ orders of magnitude larger crosssections compared to photoionization.

In contrast to the static case, if the Sun is moving, then from the upwind direction fresh molecular (neutral) hydrogen material of the IC is permanently approaching the solar system, and thus the extent $L_{\mathrm{c}}$ of the dissociation (ionization) cavity in upwind direction is defined as the upwind distance where the rate of freshly convection-imported hydrogen molecules (atoms) equals the local photodissociation (photoionization) rate. This, evidently, leads to the following expression for $L_{\mathrm{c}}$ (the index c corresponding to the case of molecular $m=$ molecular, and to the case of atomic $n=$ neutral atoms, respectively, (see for analogy Fahr 2003):

$$
L_{\mathrm{c}} \simeq \frac{4 \beta_{0, c} r_{0}^{2}}{3 V_{\mathrm{H}}},
$$

where $\beta_{0, m}\left(\beta_{0, n}\right)$ is the solar photodissociation (photoionization) rate at some reference distance $r_{0}$. The photoionization rate $\beta_{0, n}$ is equal to $8 \times 10^{-8} \mathrm{~s}^{-1}$ at the reference point $r_{\mathrm{E}}=1 \mathrm{AU}$ (Fahr et al. 2000). The dissociation of molecular hydrogen is complicated due to different possible branches of solar photon interaction with $\mathrm{H}_{2}$ molecules: the direct photodissociation rate is $3 \times 10^{-7} \mathrm{~s}^{-1}$ at $1 \mathrm{AU}$ for the moderate solar activity conditions, while the rates for photoionization of $\mathrm{H}_{2}$ and photodissociation with ionization are by order of magnitude less than the dominant direct photodissociation rate (Gruntman 1996). Thus one finds $L_{m} \simeq 2.4 \mathrm{AU}$ and $L_{n} \simeq 0.61 \mathrm{AU}$ for the cases of molecular and atomic flow, respectively. Hence the state of the material when arriving at the Earth's orbit is essentially not changed with respect to the interstellar conditions. A treatment of molecular hydrogen as a new fluid in addition to atoms and ions will be addressed in a forthcoming paper, so below we will assume that the flow is initially completely atomic at infinity.

A second ionization source may perhaps merit some brief consideration in this context, namely energetic neutral $\mathrm{H}$-atoms coming radially outwards from the inner heliosphere as $\mathrm{keV}-$ energetic transcharged solar wind protons. In fact interstellar $\mathrm{H}$-atoms penetrating into the inner supersonic solar wind region there undergo charge exchange processes with solar wind protons. This leads to the phenomenon of the neutralized solar wind as discussed by Blum \& Fahr (1970); Gruntman et al. (1990); Bleszynski et al. (1992); Gruntman (1994). One important influence of such neutralized solar wind protons on the approaching interstellar plasma flow was discussed already by Gruntman (1982). In our present case of strongly enhanced neutral gas densities one may thus also study the effects of such energetic atoms on the incoming interstellar $\mathrm{H}$-atoms and protons. It in fact turns out that such atoms are strongly interacting with the dense proton layer outside of the heliopause by heating protons up there. But due to the fact that proton densities of $n_{\mathrm{p}} \sim 10^{3} \mathrm{~cm}^{-3}$ are met these energetic neutrals are effectively absorbed by charge exchange with background protons over mean free paths of $\lambda_{\mathrm{ex}}=\left(n_{\mathrm{p}} \cdot \sigma_{\mathrm{ex}}\right)^{-1} \sim 0.1 \mathrm{AU}$. This means these atoms cannot penetrate deeply into the incoming medium and there contribute to the ionization of incoming IC H-atoms.

\section{Model of the neutral gas flow}

We approximate the situation as follows: let the Sun be in the origin of a system of cylindrical coordinates $(r, \zeta, z)$, and let the axis of symmetry $z$ be antiparallel to the motion of the Sun, that is the countermoving cloud's matter flows from negative $z$-values to positive ones. Such a picture schematically is illustrated in Fig. 1, where the streamlines of the interstellar neutral and ionized wind passing around the strongly reduced heliosphere are shown.

The Sun affects the circumsolar flow by means of gravitation and ionizing solar radiation. As is mentioned in Sect. 2, except for the interaction interface (within the range of less than $0.1 \mathrm{AU}$, of a few mean free paths of solar wind protons with respect to charge-exchange with IC H-atoms, at IC densities under consideration), the steady-state neutral flow is completely shielded from the direct solar wind influence (see illustration given by Fig. 1).

Effects of the hydrodynamical motion of the inflow upon the ionization state of the gas will be examined below. It should be underlined here that we have focused our consideration to a completely neutral inflow material and to IC densities of, and greater than, $1000 \mathrm{~cm}^{-3}$. This is enough to justify the hydrodynamic approximation all over in upwind side excluding the region with the heliocentric distances less than a few $0.1 \mathrm{AU}$, because at these densities the mean free path of the particles is less than 0.1 AU and Knudsen numbers are of the order of $K n \sim t_{\text {hydro }} / t_{\text {coll }} \sim 10$, where $t_{\text {hydro }}$ and $t_{\text {coll }}$ are the characteristic hydrodynamic and collision times, respectively.

To describe the behavior of the flow one can choose a twofluid model of hydrogen atoms and protons, which is an extension of one-fluid model of Whang (1996) and Yeghikyan $\&$ Fahr (2003). Therefore a set of conservation laws for massmomentum-energy-flows with the right-sides containing corresponding source terms due to gravitation, photoionization and the mutual charge-exchange, can be written in the form

$$
\begin{aligned}
& \frac{\partial \rho_{j}}{\partial t}+\nabla\left(\rho_{j} \boldsymbol{V}_{j}\right)=S_{\rho, j} \\
& \frac{\partial\left(\rho_{j} \boldsymbol{V}_{j}\right)}{\partial t}+\nabla \boldsymbol{\Pi}_{j}=\rho_{j}\left(1-\mu_{j}\right) \nabla\left(\frac{G M}{R}\right)+S_{\mathrm{V}, j} \\
& \frac{\partial E_{j}}{\partial t}+\nabla\left[\boldsymbol{V}_{j}\left(E_{j}+P_{j}\right)\right]=\rho_{j}\left(1-\mu_{j}\right) \boldsymbol{V}_{j} \nabla\left(\frac{G M}{R}\right)+S_{\mathrm{E}, j}
\end{aligned}
$$

Here $\rho_{j}, \boldsymbol{V}_{j}$ and $P_{j}$ are density, velocity and scalar pressure of the species $j=\mathrm{n}$ (neutral hydrogen) and $j=\mathrm{p}$ (protons), 
respectively, $\boldsymbol{\Pi}_{l k, j}=\rho_{j} V_{l} V_{k}+P_{j} \delta_{l k}$, and $E_{j}=\frac{1}{2} \rho_{j} V_{j}^{2}+\frac{P_{j}}{\gamma-1}$ are the HD-stress tensor and the total energy per unit volume, respectively, $R=\sqrt{\left(r^{2}+z^{2}\right)}$ is the solar distance in a cylindrical coordinate system $(r, \zeta, z)$ with symmetry axis $z, \gamma=5 / 3$ is the ratio of specific heats for monatomic hydrogen gas, $\mu_{j}$ is a factor denoting the ratio of the repulsion force by solar $L_{\alpha}$ radiation to the gravitational attraction force $\left(\mu_{j}=0\right.$ for $\left.j=\mathrm{p}\right)$ and $M$ and $G$ are the solar mass and the gravitational constant, respectively.

The source terms in Eqs. (7)-(9) result from the interaction of the flow components with the gravitational and radiational fields of the Sun and in addition from their mutual conversion due to charge-exchange. Taking all of this together leads to the following source terms:

$S_{\rho, \mathrm{n}}=-\beta_{\mathrm{phi}} \rho_{\mathrm{n}}+\rho_{\mathrm{p}}^{2} \alpha / m_{\mathrm{p}}$,

$S_{\rho, \mathrm{p}}=\beta_{\mathrm{phi}} \rho_{\mathrm{n}}-\rho_{\mathrm{p}}^{2} \alpha / m_{\mathrm{p}}$,

$S_{V, \mathrm{n}}=-\beta_{\mathrm{phi}} \rho_{\mathrm{n}} \boldsymbol{V}_{\mathrm{n}}-v_{\mathrm{ce}}^{-} \rho_{\mathrm{n}}\left(\boldsymbol{V}_{\mathrm{n}}-\boldsymbol{V}_{\mathrm{p}}\right)+\rho_{\mathrm{p}}^{2} \alpha / m_{\mathrm{p}}$,

$S_{\boldsymbol{V}, \mathrm{p}}=\beta_{\mathrm{phi}} \rho_{\mathrm{n}} \boldsymbol{V}_{\mathrm{n}}+v_{\mathrm{ce}}^{-} \rho_{\mathrm{n}}\left(\boldsymbol{V}_{\mathrm{n}}-\boldsymbol{V}_{\mathrm{p}}\right)-\rho_{\mathrm{p}}^{2} \alpha / m_{\mathrm{p}}$,

$S_{E, \mathrm{n}}=-\beta_{\mathrm{phi}} E_{\mathrm{n}}+v_{\mathrm{ce}}^{-}\left(E_{\mathrm{p}}-E_{\mathrm{n}}\right)$,

$S_{E, \mathrm{p}}=\beta_{\mathrm{phi}} E_{\mathrm{n}}-v_{\mathrm{ce}}^{+}\left(E_{\mathrm{p}}-E_{\mathrm{n}}\right)$,

where charge-exchange collision rates of hydrogen atoms and protons are given by $\nu_{\mathrm{ce}}^{-}=\sigma_{\mathrm{ce}} \cdot v_{\mathrm{rel}} n_{\mathrm{p}}$ and $\nu_{\mathrm{ce}}^{+}=\sigma_{\mathrm{ce}} \cdot v_{\mathrm{rel}} n_{\mathrm{n}}$, respectively, $v_{\text {rel }}=\sqrt{3.76 \times 10^{8}\left(T_{\mathrm{p}}+T_{\mathrm{n}}\right)+\left(\boldsymbol{V}_{\mathrm{n}}-\boldsymbol{V}_{\mathrm{p}}\right)^{2}}$, (in $\mathrm{cm} / \mathrm{s}), \sigma_{\text {ce }}=\left[2.1 \times 10^{-7}-9.2 \times 10^{-9} \ln \left(v_{\text {rel }}\right)\right]^{2}\left(\sigma_{\text {ce }}\right.$ in $\left.\mathrm{cm}^{2}\right), n_{\mathrm{p}}$ and $n_{\mathrm{n}}$ are number density for protons and neutral atoms, respectively (Fahr et al. 2000), and $\alpha=4.0 \times 10^{-10} / T_{\mathrm{p}}^{0.8}$ (e.g. Osterbrock 1989) is a photorecombination coefficient in a recombination process of protons with electrons (assumed that $\left.n_{\mathrm{p}} \sim n_{\mathrm{e}}\right)$.

The ionization rate of neutral hydrogen atom by photoionization for optically thin case is approximately defined as

$\beta_{\mathrm{phi}}=\beta_{0}\left(\frac{r_{\mathrm{E}}}{R}\right)^{2}$,

with $\beta_{0}=\beta_{0, \mathrm{phi}}=8 \times 10^{-8} \mathrm{~s}^{-1}$, that is by about one order of magnitude less than the ionization rate due to charge exchange with solar protons (i.e. inside the heliosphere), $\beta_{0 \text {,ce, which is }}$ equal to $7.68 \times 10^{-7} \mathrm{~s}^{-1}$ at the same reference point $r_{\mathrm{E}}$ (Fahr et al. 2000). In the following we will use $\beta_{0}=\beta_{0, \text { phi }}$ according to the above condition that neutral flow material in the region described in this paper is completely shielded from solar wind protons.

The flow is assumed to be symmetrical with respect to the $z$-axis, so the results shown here can be rotated by an azimuthal angle $\zeta$ with respect to the $z$-axis.

For an axis-symmetrical case, when all derivatives in $\zeta$ vanish, using dimensionless variables and omitting " $j$ indices", we introduce

$\tilde{\rho}=\rho / \rho_{i}, \tilde{u}=u / V_{i}, \tilde{v}=v / V_{i}, \tilde{P}=P / \rho_{i} V_{i}^{2}$,

$\tilde{E}=E / E_{i}, \tilde{t}=t V_{i} / \lambda, \lambda=\beta_{0} r_{\mathrm{E}}^{2} / V_{i}, \tilde{z}=z / \lambda, \tilde{r}=r / \lambda$,

where $u$ and $v$ are the corresponding components of velocity $\boldsymbol{V}$. This system has a standard vector form of a hyperbolic set of partial differential equations (LeVeque et al. 1998) with relevant source terms on the right sides given in the form:

$\boldsymbol{q}_{t}+\boldsymbol{A}(\boldsymbol{q})_{z}+\boldsymbol{B}(\boldsymbol{q})_{r}=\boldsymbol{\Psi}(r, z, t, \boldsymbol{q})$.

The explicit expressions for the $\boldsymbol{A}, \boldsymbol{B}$ and $\boldsymbol{\Psi}$ and other details can be found in Yeghikyan \& Fahr (2003).

The initial data describe an inner cavity with shape given by Eq. (5), and the neutral flow number density from Fahr (1968a). The boundary conditions are treated like in Fahr et al. (2000), that is an inflow of completely neutral material from the left boundary, the bottom boundary (i.e. the axis) is a "reflecting" boundary by symmetry reasons and the top and right boundaries are outflow boundaries (zero-order extrapolation). We have limited our calculations here to the upwind side only, up to the reduced heliosphere, before the heliopause: it should be born in mind that the condition inside the heliosphere has no influence on the supersonic flow outside in this region.

With the adopted values $\rho_{\mathrm{E}} / m_{\mathrm{p}}=5 \mathrm{~cm}^{-3}, \rho_{\mathrm{i}} / m_{\mathrm{p}}=$ $1000 \mathrm{~cm}^{-3}$ ( $m_{\mathrm{p}}$ is the proton mass), $V_{\mathrm{w}}=400 \mathrm{~km} \mathrm{~s}^{-1}$ and $V_{\mathrm{i}}=26 \mathrm{~km} \mathrm{~s}^{-1}$, we have solved the above system by means of the LeVeque's code CLAWPACK (LeVeque et al. 1998), based on Godunov's method with high-order correction terms, on a uniform grid with $160 \times 60$ points. Here we present results for the most important cases with IC densities $n_{\mathrm{i}} \sim 1000 \mathrm{~cm}^{-3}$ and temperatures $T_{\mathrm{i}} \simeq 100 \mathrm{~K}$ as shown in Figs. 2 and 3. Our choice of $T_{\mathrm{i}}$ is based on the fact that the dynamical timescale $\tau_{\mathrm{d}} \sim D / V_{\mathrm{H}} \sim 20 \mathrm{yr}$ is much less than a photoionization timescale $\tau_{\text {phi }}$, which can be defined as $1 / \beta \sim 1 / \beta_{\text {phi }} / D^{2} \sim$ $4000 \mathrm{yr}$, where $D \sim 100 \mathrm{AU}$ is the characteristic dimension. In turn $\tau_{\mathrm{phi}}$ is less than any timescale, related with an injection rate of energy into the flow, far from the Sun in the upwind direction, and caused, apparently only by photoionization.

Length units are in units of the ionizational length scale $\lambda$, which is equal to $0.46 \mathrm{AU}$ for the adopted parameters, and for the used calculational domain this corresponds to a spatial resolution of $0.2 \mathrm{AU}$ along the $z$-axis. All model calculations have been carried out as time proceeds until asymptotically a steady configuration is reached (corresponding to a few hundred years in real time).

In Fig. 2 the iso-density and iso-velocity contours of hydrogen atoms and protons as functions of coordinates $r$ and $z$ are shown, while in Fig. 3 the distributions of density and axial components of velocity are represented versus axial distanse $z$ at the corresponding radial coordinate $r=0$. Transverse velocity components due to symmetry reasons disappear at the axis. For that reason we display these components at an axis-line shifted by $1 \mathrm{AU}$ (that is at $r=1 \mathrm{AU}$ and $z_{\min } \leq z \leq z_{\max }$ ).

In Fig. 3 we compare the calculated distribution of density for different (gravitating, not-gravitating) cases with that of cold (collisionless) kinetic model (e.g. see Holzer 1977, his Eq. (21)) for the same input parameters and $\beta=\beta_{\text {phi }}=8 \times 10^{-8} \mathrm{~s}^{-1}$. The density decrease (increase) for the atoms (protons) close to the Sun is evidently caused by the photoionization, while the role of the gravity is visible only inside the region, limited by a so called accretion radius $r_{\mathrm{g}}=2 G M / V_{\mathrm{i}}^{2}$ (Hunt 1971) (cf. the cases $G \neq 0$ and $G=0$ in Fig. 3). A good coincidence of the density distribution in the cold kinetic and 

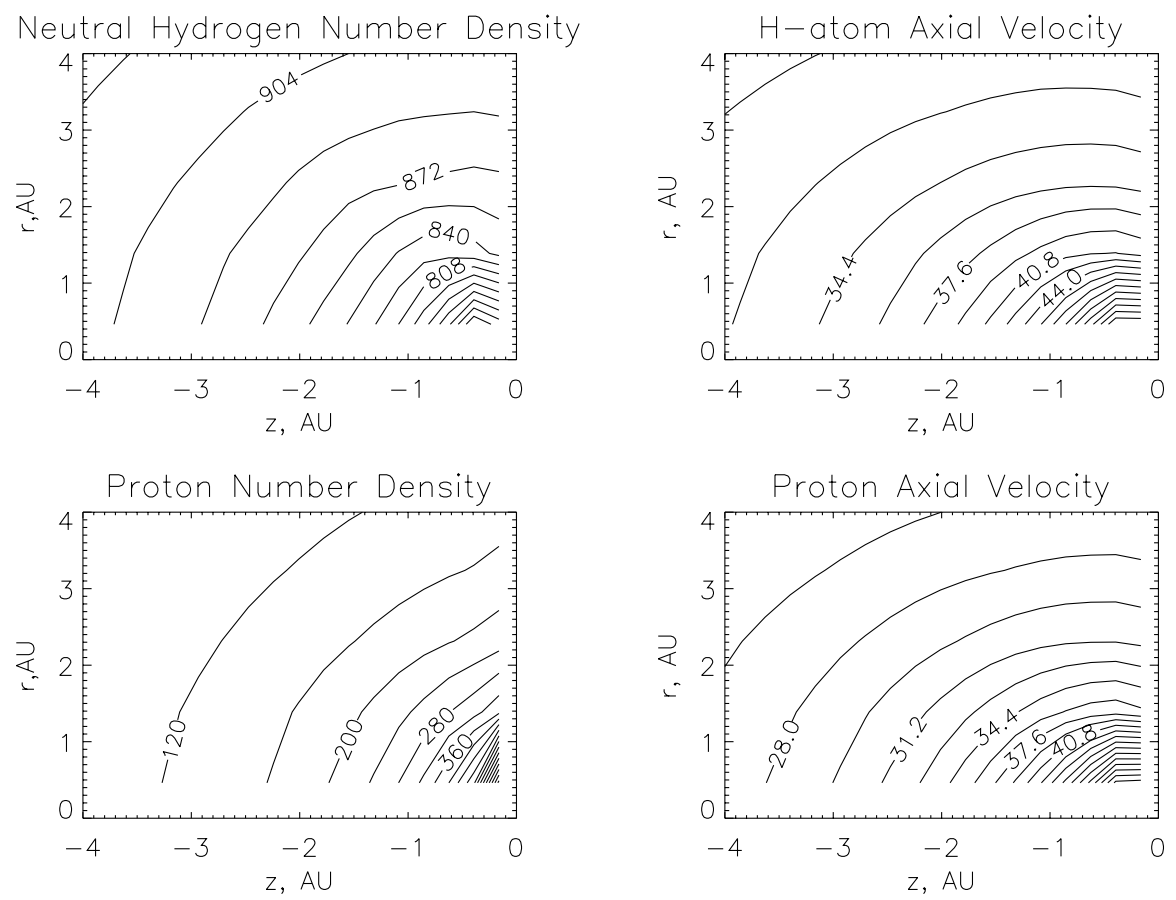

Fig. 2. Iso-density and axial velocity contours for the hydrogen atoms and protons in the $r-z$ plane. Contours are labeled by values in units $\mathrm{cm}^{-3}$ and $\mathrm{km} \mathrm{s}^{-1}$ respectively. Values of the input parameters are: $n_{\mathrm{i}}=1000 \mathrm{~cm}^{-3}, V_{\mathrm{i}}=26 \mathrm{~km} \mathrm{~s}^{-1}, T_{\mathrm{i}}=100 \mathrm{~K} . \beta_{0}=\beta_{\text {phi }}, \mu=0.1$.
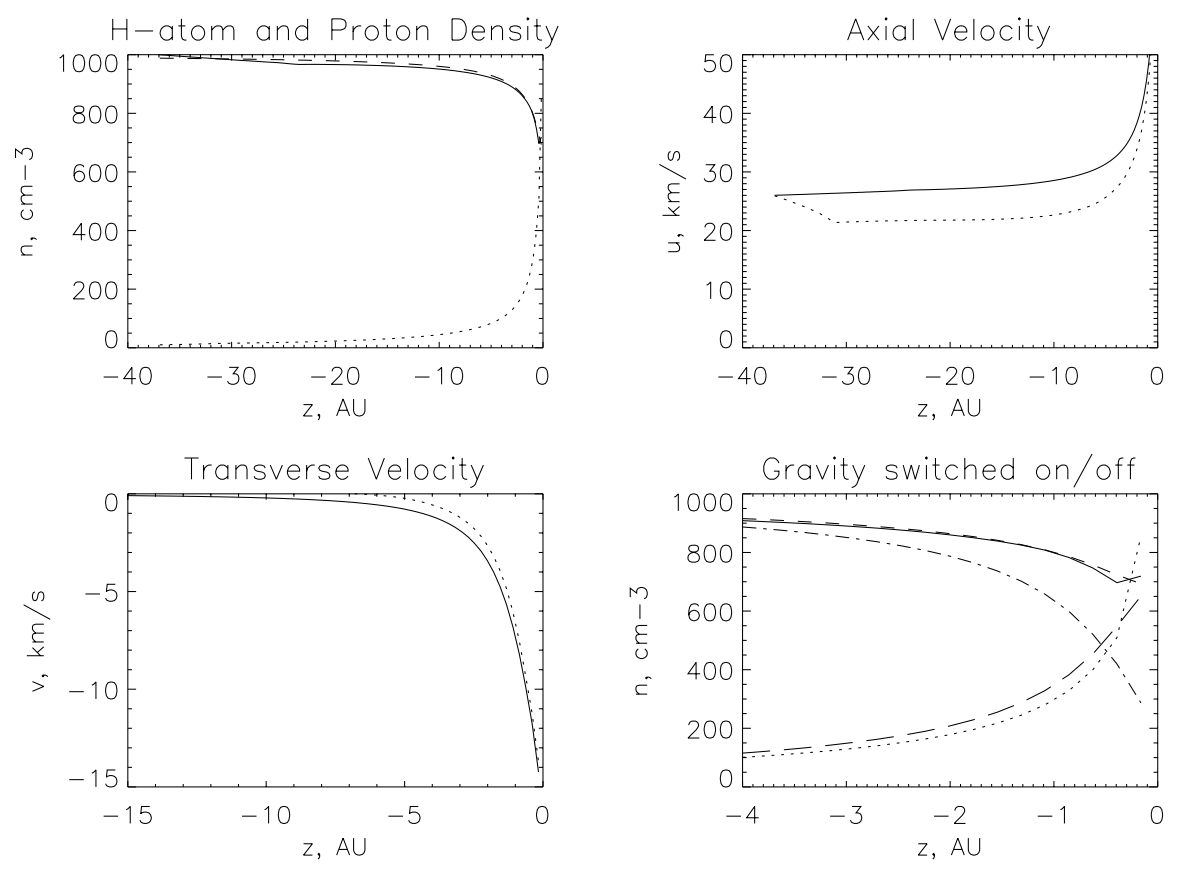

Fig. 3. Number densities of the IC H-atom and proton flow versus axial distanse $z$, at radial coordinate $r=0$ (transverse velocities are shown at $r=1 \mathrm{AU})$. Solid and dotted lines are used to distinguish between atoms and protons, respectively. $n_{\mathrm{i}}=1000 \mathrm{~cm}^{-3}, V_{\mathrm{i}}=26 \mathrm{~km} \mathrm{~s}$, $T_{\mathrm{i}}=100 \mathrm{~K} . \beta_{0}=\beta_{\mathrm{phi}}, \mu=0.1$, the spatial resolution along the $z$-axis is $0.2 \mathrm{AU}$. The role of gravity is illustrated by the presence and absence of the gravitational field of the Sun, shown in the bottom, right, by solid and dotted (for atoms and protons, $G \neq 0$ ) and by dash-dotted and long-dashed lines (for atoms and protons, $G=0$ ), respectively. The density distributions in the cold kinetic model (see text) with the same input parameters are also shown in the top, left and bottom, right, by the dashed lines.

hydrodynamic models justifies our hydrodynamic approach in the calculational domain.

The optical depth in the $L y-\alpha$-hydrogen line even at the 1 AU would always be greater than 1 for $n_{\mathrm{i}} \simeq 100 \mathrm{~cm}^{-3}$. At the same time a contribution of diffusely scattered $L y-\alpha$ photons to the repulsion force would be nearly negligible. The case is that only the first scattering of the solar $L y-\alpha$ photon contributes to the radiation pressure, because multiply scattered photons are more or less isotropic and thus do not contribute significantly to the momentum transfer anymore - the net effect thus 
would be nearly zero. The solar $L y-\alpha$ profile has about $1 \AA$ FWHM width, whereas the IC $\mathrm{H}$ atom of the flow with a radial velocity component of $40 \mathrm{~km} \mathrm{~s}^{-1}$ would scatter photons, shifted only of $0.2 \AA$ from a center of the line (Holzer 1977). It evidently means that the distant regions of the flow with respect to the Sun will not receive direct solar $L y-\alpha$ radiation due to absence of any considerable velocity gradient and consequent absorption. Thus $\mu=0.1$ seems to be an appropriate approximation.

It is clear that only when $R \geq r_{\mathrm{g}}$, the flow is unaffected by gravitation (Talbot \& Newman 1977). For $V_{\mathrm{i}}=26 \mathrm{~km} \mathrm{~s}^{-1}$ one finds $r_{\mathrm{g}}=2.6 \mathrm{AU}$. It means, that the case of Bondi's flow with $\rho \simeq \rho_{\mathrm{i}}\left(r_{\mathrm{g}} / R\right)^{3 / 2}$ and $V^{2} \simeq 2 G M / R$ is applicable inside the distance $r_{\mathrm{g}}$ (see Fig. 3).

We can now estimate the amount of neutral gas, accreted by the Earth (an interaction between the charged particles and the magnetosphere is more complicated, and we simply assume here, that all of them will be deflected). By order of magnitude accuracy one may write for the amount of accreted matter (Butler et al. 1978): $\mathrm{d} M / \mathrm{d} t \simeq \pi R_{\mathrm{E}}^{2} \cdot V_{\text {rel }} \cdot n \cdot m_{\mathrm{p}}\left(\mathrm{g} \mathrm{s}^{-1}\right)$, where $R_{\mathrm{E}}$ is the Earth's radius, which practically coincides with the Earth's accretion radius at relative velocity of the $\mathrm{H}$-atom with respect to the Earth $V_{\text {rel }}$ of about $40 \mathrm{~km} \mathrm{~s}^{-1}$. It is clear that there is an annual variation in $V_{\text {rel }}$ between 10 to $70 \mathrm{~km} \mathrm{~s}^{-1}$ due to the orbital velocity of the Earth, equal to about $30 \mathrm{~km} \mathrm{~s}^{-1}$. An average value of $40 \mathrm{~km} \mathrm{~s}^{-1}$ therefore would be quite reasonable. Due to the many uncertainties involved in the downstream region, where the direct influence of the solar wind protons is essential we have used an upstream density value at $1 \mathrm{AU}$. For example, for $n_{\mathrm{i}} \simeq 1000 \mathrm{~cm}^{-3}$, using calculated values of $n$ at $1 \mathrm{AU}$ (about $800 \mathrm{~cm}^{-3}$, see Figs. 2 and 3), one finds that during the pass-over time of about 1 million years the Earth may accrete about $2 \times 10^{17} \mathrm{~g}$ per one passage.

The calculated value $n$ by one order of magnitude less than estimated in Butler et al. (1978), because lower relative velocities of the encounter (about $2 \mathrm{~km} \mathrm{~s}^{-1}$ with corresponding $r_{\mathrm{g}} \sim 400 \mathrm{AU}$ ) and the case without photoionization of the neutral flow were considered.

It should be highlighted here that nowhere in the heliosphere exists a sink mechanism for neutral atoms other than photoionization (electron impact ionization at $1 \mathrm{AU}$ would be negligible). Newly created fast neutrals (due to charge exchange with solar wind protons) also may be accreted by the Earth. The quantitative investigation of such model will be addressed in a forthcoming paper.

A responce of the terrestrial atmosphere to such an amount of accreted neutral hydrogen gas may be essential. Neutral hydrogen fluxes at the Earth would increase up to $10^{10}$ atoms $/ \mathrm{sm}^{2} / \mathrm{s}\left(n_{\mathrm{H}}=10^{3} \mathrm{~cm}^{-3}\right)$, which is greater by 4 orders of magnitude than at present. This highly reactive hydrogen could reach stratospheric heights at $40-50 \mathrm{~km}$ by direct inflow and accumulate to levels that may be relevant for oxygen locking, ozone depletion etc., and inevitably cause strong influences to the terrestrial atmosphere in general, and to the terrestrial climate in particular (Fahr 1968a,b; Bzowski et al. 1996; Frisch et al. 2002).

Besides Yabushita \& Allen (1983) have argued that if the Sun should ever have passed through the dense core of a GMC
$\left(n_{\mathrm{H}}=10^{5} \mathrm{~cm}^{-3}\right)$, then as much oxygen as is now present in the terrestrial atmosphere could have been removed due to water formation. In the recent paper Yabushita \& Allen (1997) have pointed out that the reduced abundance of oxygen indicated by oxygen contained in ancient amber may be a record of such an event 65 Myr ago.

Finally, one can show (Yeghikyan \& Fahr 2003a) that in the case of a countermoving GMC with usual density of $n_{\mathrm{H}}=$ $10^{3} \mathrm{~cm}^{-3}$ there would be a considerable decrease of the ozon concentration in the mesosphere.

\section{Conclusions}

In this paper we have discussed a problem of passages of the Solar System through dense interstellar gas clouds with non negligible dust content. We have shown that such clouds increase the inflow density and push the heliopause down to the Earth with the result that counterstreaming IC material directly influences the Earth. In contrast to the static case, when the Sun is moving, then from the upwind direction fresh molecular (neutral) hydrogen material of the IC is permanently approaching the solar system, and thus the extent of the dissociation (ionization) cavity in upwind direction is defined as a distance where the rate of freshly imported hydrogen molecules (atoms) equals the local photodissociation (photoionization) rate. It is shown that close to the Sun, at $1 \mathrm{AU}$, the cloud's matter is predominantly neutral and is completely shielded from the direct solar wind influence. At IC H-atom densities of the order of $n_{\mathrm{H}}=10^{3} \mathrm{~cm}^{-3}$ and more, incoming matter when approaching the Sun, but yet outside the reduced heliopause, is only subject to photoionization processes which simplify the hydrodynamic model of the flow.

We have used a simple two-fluid treatment of the incoming flow, ionized by the solar UV, and a mutual conversion of hydrogen atoms and protons due to charge exchange process has been taken into account. The time-dependent simulation of flow parameters we have carried out by means of the 2D-gasdynamic numerical code which takes into account both photoionization and gravity of the Sun, as well as the mentioned charge-exchange process. These processes are important to quantitatively estimate the total amount of neutral material, accreted from the cloud by the Earth during the pass-over time of about 1 million years. We have estimated this amount to be greater than $2 \times 10^{17} \mathrm{~g}$ per 1 passage. About $1 \%$ of which is in the form of dust $\left(\mathrm{H}_{2} \mathrm{O}\right.$ "dirty" ice or silicate grains, or both) with probable refractory organic species in the mantle (Yeghikyan et al. 2001). It may be important as a source of accumulated prebiotic material on the Earth during manyfold passages of the Solar System through similar interstellar clouds.

Terrestrial atmospheric responce to the inflow of the greatly enhanced fluxes of neutral hydrogen atoms may be considerable and definitely worthwhile to be studied. An appropriate discussion is presented separately (Yeghikyan \& Fahr 2003a).

Acknowledgements. A. Y. was supported financially by the Alexander von Humboldt Foundation, and by the Institute of Astrophysics and Extraterrestrial Research, University of Bonn. Financial support by the Deutsche Forschungsgemeinschaft in the frame of the project 
"Helio-trigger" (Fa 97/28-1) is also gratefully acknowledged. The authors thank K. Scherer for critical comments and our referee, S. Grzedzielski for constructive review comments.

\section{References}

Allen's Astrophysical Quantities 2000, ed. A. N. Cox (Springer)

Baranov, V. B., Krasnobaev, K. V, \& Kulikovsky, A. G. 1971, Sov. Phys. Dokl., 15, 791

Baranov, V. B., \& Malama, Yu. G. 1993, J. Geophys. Res., 98, 15157

Bleszynski, S., Grzedzielski, S., Rucinski, D., \& Jakimiec, J. 1992, Planet. Space Sci., 40, 1525

Blum, P. W., \& Fahr, H. 1970, A\&A, 4, 280

Butler, D. M., Newman, M. J., \& Talbot, Jr. R. J. 1978, Science, 201, 522

Bzowski, M., Fahr, H.-J., \& Rucinski, D. 1996, Icarus, 124, 209

Fahr, H. J. 1968a, Ap\&SS, 2, 474

Fahr, H. J. 1968b, Ap\&SS, 2, 496

Fahr, H. 2000, The Outer Heliosphere: Beyond the Planets, ed. K. Scherer, H. Fichtner, \& E. Marsch (Copernicus, KatlenburgLindau), 67

Fahr, H. J. 2002, Ann. Geophys., 20, 1509

Fahr, H. -J. 2003, Adv. Space Res., in press

Fahr, H. J., \& Rucinski, D. 2002, Nonlinear Proc. Geophys., 9, 377

Fahr, H. J., \& Chashei, I. 2002, A\&A, 395, 991

Fahr, H. J., Ripken, H. W., \& Lay, G. 1981, A\&A, 102, 359

Fahr, H. J., Grzedzielski, S., \& Ratkiewicz, R. 1988, Ann. Geophys., 6,337

Fahr, H. J., Kausch, T., \& Scherer, H. 2000, A\&A, 357, 268

Frisch, P. C., Muller, H. R., Zank, G. P., \& Lopate C. 2002 [astro-ph/0208556]

Frisch, P. C. 1996, Space Sci. Rev., 78, 213

Gruntman, M. 1982, Sov. Astron. Lett., 8, 24

Gruntman, M. 1994, J. Geophys. Res., 99, 19213

Gruntman, M. 1996, J. Geophys. Res., 101, 15555

Gruntman, M., Leonas, V. B., \& Grzedzielski, S., Physics of the outer heliosphere, ed. S. Grzedzielski, \& D. E. Page (NY: Pergamon Press), 355, 1990

Guinan, E. F., \& Ribas, I. 2002, The evolving Sun and its influence on planetary environments, ASP Conf. Ser., 269, 85

Heiles, C., Goodman, A. A., McKee, C. F., \& Zweibel, E. G. 1991, Fragmentatation of molecular clouds and star formation, ed. E. Falgarone, F. Boulander, \& G. Duvert (Kluwer AP), IAU Symp., 147, 43

Holzer, T. E. 1977, Rev. Geophys. Space Phys., 15, 467
Hunt, R. 1971, MNRAS, 154, 141

Krasnobaev, K. V. 1971, Sov. Ast., 14, 840

Lammer, H., Stumptner, W., Molina-Cuberos, G. J, Lara, L. M., \& Tehrany, M. G. 2002, The evolving Sun and its influence om planetary environments, ASP Conf. Ser., 269, 85

LeVeque, R. J. 1998, in Computational methods for astrophysical fluid flow, Saas-Fee Advanced Course 27, ed. R. J. LeVeque, D. Mihalas, E. A. Dorfi, \& E. Muller (Springer)

McKee, C. F. 1995, The Physics of the Interstellar medium and Intergalactic Medium, ed. A. Ferrara, C. F. McKee, C. Heiles, \& P. R. Shapiro, ASP Conf. Ser., 80, 292

Osterbrock, D. E. 1989, Astrophysics of Gaseous Nebulae and Active Galactic Nuclei (Mill Valley: University Science Books)

Parker, E. N. 1963, Interplanetary dynamical processes (Interscience Publishers)

Pauls H. L., Zank, G .P., \& Williams, L. L. 1995, J. Geophys. Res., 100,21595

Ratkiewicz, R. 1992, A\&A, 255, 383

Ripken, H. W., \& Fahr H. J. 1981, Solar Wind Four Conf. Burghausen, Aug. 28, 1978, 528

Sackmann, I., \& Boothroyd, A. I., Our Sun. V. 2003, ApJ, 583, 1024

Scherer, K. 2000, The Outer Heliosphere: Beyond the Planets, ed. K. Scherer, H. Fichtner, \& E. Marsch (Copernicus, KatlenburgLindau), 327

Scherer, K., Fichtner, H., \& Stawicki, O. 2002, J. Atmosph. Solar-Terr. Phys., 64, 795

Spitzer, L., Jr. 1978, Physical Processes in the Interstellar Medium (A Wiley-Int. Publ.)

Talbot, R. J., Jr., \& Newman, M. J. 1977, ApJS, 34, 295

Whang, Y. C. 1996, ApJ, 468, 947

Wilkin, F. P. 1996, ApJ, 459, L31

Williams, R. E. 1965, ApJ, 142, 314

Wimmer-Schweingruber, R. F., \& Bochsler, P. 2000, Acceleration and Transport of Energetic Particles Observed in the Heliosphere: ACE 2000 Symp., ed. R. A. Mewaldt et al., 270

Yabushita, S., \& Allen, A. J. 1983, Observatory, 103, 249

Yabushita, S., \& Allen, A. J. 1985, Observatory, 105, 198

Yabushita, S., \& Allen, A. J. 1989, MNRAS, 238, 1465

Yabushita, S., \& Allen, A. J. 1997, Astron. Geophys., 38, 15

Yeghikyan, A. G., \& Fahr, H. J. 2003, Ann. Geophys., 21, 1263

Yeghikyan, A. G., \& Fahr, H. J. 2003a, A\&A, submitted

Yeghikyan, A. G., Viti, S., \& Williams, D. A. 2001, MNRAS, 326, 313

Zahnle, K. J., \& Walker, J. C. G. 1982, Rev. Geophys. Space Phys., 20,280

Zank, G. P., \& Frisch, P. C. 1999, ApJ, 518, 965 